\title{
Training Motor Skills Using Haptic Interfaces
}

\author{
Otniel Portillo-Rodriguez ${ }^{1,2}$, Carlo Avizzano1, \\ Oscar Sandoval Gonzalez ${ }^{1,3}$, Adriana Vilchis-Gonzalez², \\ Mariel Davila-Vilchis ${ }^{2}$ and Massimo Bergamasco ${ }^{1}$ \\ 1 Perceptual Robotics Laboratory, Scuola Superiore Sant'Anna, Pisa, \\ ${ }^{2}$ Facultad de Ingeniería, Universidad Autónoma del Estado de México, Toluca, \\ Instituto Tecnológico de Orizaba, Orizaba, \\ ${ }^{1}$ Italy \\ 2,3 México
}

\section{Introduction}

Skill has many meanings, as there are many talents: its origin comes from the late Old English scele, meaning knowledge, and from Old Norse skil (discernment, knowledge), even if a general definition of skill can be given as "the learned ability to do a process well" (McCullough, 1999) or as the acquired ability to successfully perform a specific task.

Task is the elementary unit of goal directed behaviour (Gopher, 2004) and is also a fundamental concept -strictly connected to "skill"- in the study of human behaviour, so that psychology may be defined as the science of people performing tasks. Moreover skill is not associated only to knowledge, but also to technology, since technology is -literally in the Greek- the study of skill.

Skill-based behaviour represents sensory-motor performance during activities following a statement of an intention and taking place without conscious control as smooth, automated and highly integrated patterns of behaviour. As it is shown in Figure 1, a schematic representation of the cognitive-sensory-motor integration required by a skill performance, complex skills can involve both gesture and sensory-motor abilities, but also high level cognitive functions, such as procedural (e.g. how to do something) and decision and judgement (e.g. when to do what) abilities. In most skilled sensory-motor tasks, the body acts as a multivariable continuous control system synchronizing movements with the behavioural of the environment (Annelise Mark Pejtersen, 1997). This way of acting is also named also as, action-centred, enactive, reflection-in-action or simply know-how.

Skills differ from talent since talent seems native, and concepts come from schooling, while skill is learned by doing (McCullough, 1999). It is acquired by demonstration and sharpened by practice. Skill is moreover participatory, and this basis makes it durable: any teacher knows that active participation is the way to retainable knowledge.

The knowledge achieved by an artisan throughout his/her lifelong activity of work is a good example of a skill that is difficult to transfer to another person. At present the knowledge of a specific craftsmanship is lost when the skilled worker ends his/her working 
activity or when other physical impairments force him/her to give up. The above considerations are valid not only in the framework of craftsmanship but also for more general application domains, such as the industrial field, e.g. for maintenance of complex mechanical parts, surgery training and so on.

The research done stems out from the recognition that technology is a dominant ecology in our world and that nowadays a great deal of human behaviour is augmented by technology. Multimodal Human-Computer Interfaces aim at coordinating several intuitive input modalities (e.g. the user's speech and gestures) and several intuitive output modalities.

The existing level of technology in the HCI field is very high and mature, so that technological constrains can be removed from the design process to shift the focus on the real user's needs, as it is demonstrated by the fact that nowadays the user-centered design has became fundamental for devising successful everyday new products and interfaces (Norman, 1986; Norman, 1988), fitting people and that really conforming their needs.

However, until now most interaction technologies have emphasized more input channel (afferent channel in Figure 1 The role of HCI in the performance of a skill), rather than output (efferent channel); foreground tasks rather than background contexts.

Advances in $\mathrm{HCI}$ technology allows now to have better gestures, more sensing combinations and improve 3D frameworks, and so it is possible now to put also more emphasis on the output channel, e.g. recent developments of haptic interfaces and tactile effector technologies. This is sufficient to bring in the actual context new and better instruments and interfaces for doing better what you can do, and to teach you how to do something well: so interfaces supporting and augmenting your skills. In fact user interfaces to advanced augmenting technologies are the successors to simpler interfaces that have existed between people and their artefacts for thousands of years (M. Chignell \& Takeshit, 1999).

The objective is to develop new HCI technologies and devise new usages of existing ones to support people during the execution of complex tasks, help them to do things well or better, and make them more skilful in the execution of activities, overall augmenting the capability of human action and performance.

We aimed to investigate the transfer of skills defined as the use of knowledge or skill acquired in one situation in the performance of a new, novel task, and its reproducibility by means of VEs and HCI technologies, using actual and new technology with a complete innovative approach, in order to develop and evaluating interfaces for doing better in the context of a specific task.

Figure 2 draws on the scheme of Figure 1, and shows the important role that new interfaces will play and their features. They should possess the following functionalities:

- Capability of interfacing with the world, in order to get a comprehension of the status of the world;

- Capability of getting input from the humans through his efferent channel, in a way not disturbing the human from the execution of the main task (transparency);

- Local intelligence, that is the capability of having an internal and efficient representation of the task flow, correlating the task flow with the status of the environment during the human-world interaction process, understanding and 
predicting the current human status and behaviour, formulating precise indications on next steps of the task flow or corrective actions to be implemented;

- Capability of sending both information and action consequences in output towards the human, through his/her afferent channel, in a way that is not disturbing the human from the execution of the main task.

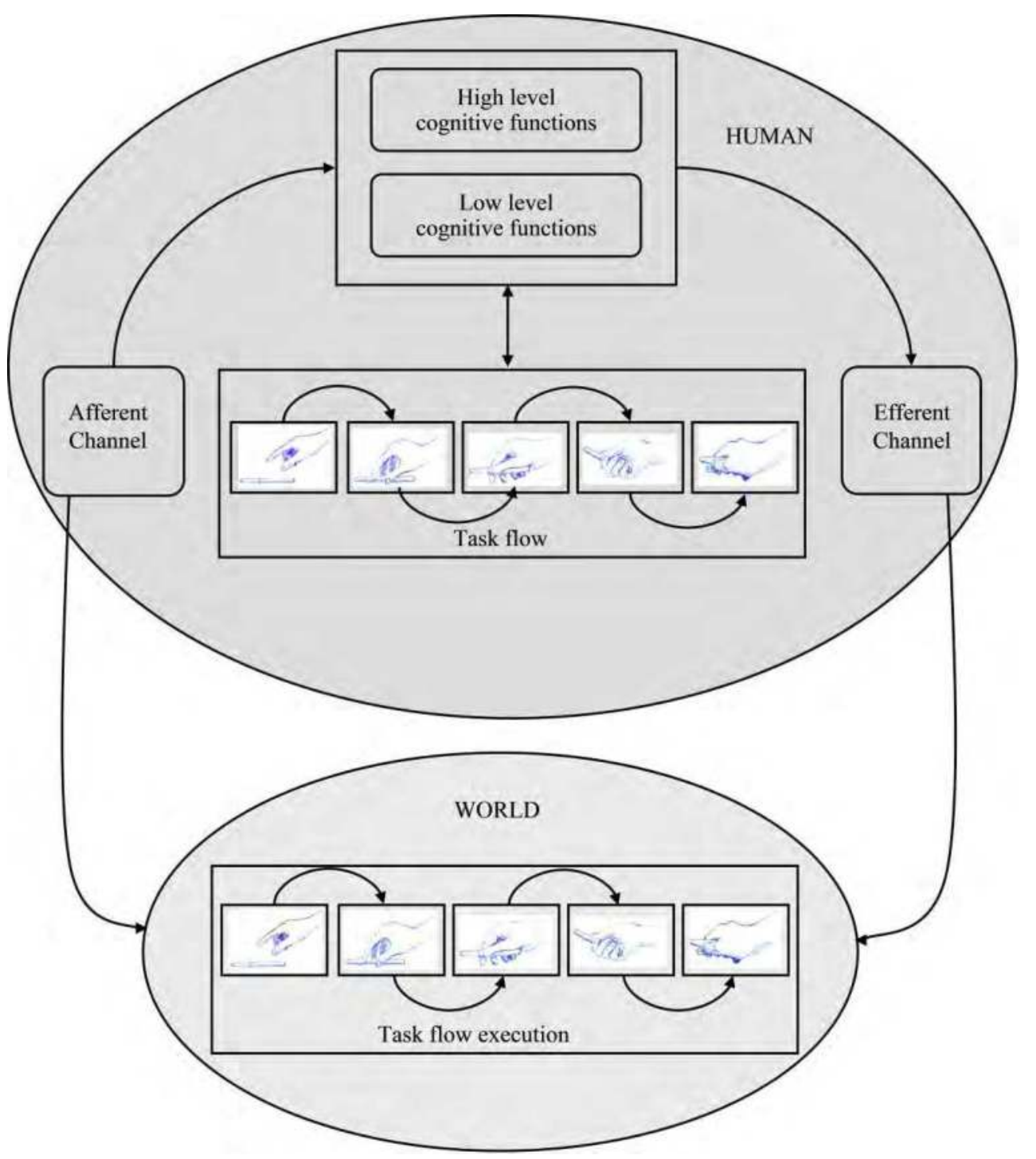

Fig. 1. A schematic representation of the cognitive-sensory-motor integration required by a skill performance 
We desire improving both input and output modalities of interfaces, and on the interplay between the two, with interfaces in the loop of decision and action (Flach, 1994) in strictly connection with human, as it is shown clearly in Figure 2. The interfaces will boost the capabilities of the afferent-efferent channel of humans, the exchange of information with the world, and the performance of undertaken actions, acting in synergy with the sensorymotor loop.

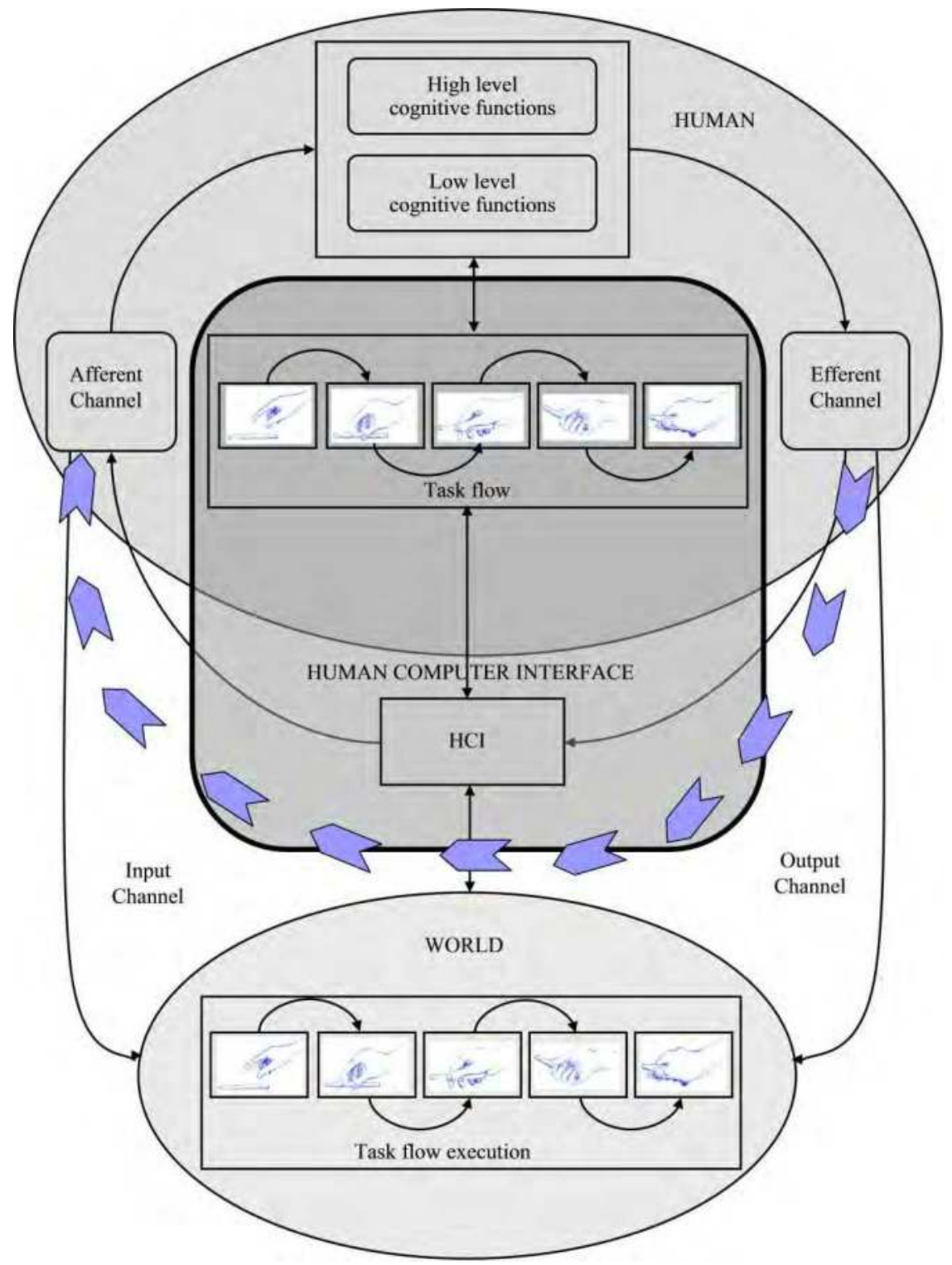

Fig. 2. The role of $\mathrm{HCI}$ in the performance of a skill 
Interfaces will be technologically invisible at their best -not to decrease the human performance-, and capable of understanding the user intentions, current behaviour and purpose, contextualized in the task.

In this chapter a proposal of one multimodal interface for the transferring of skills, it will be described. The interface is called Haptic Desktop System (HDS), it was specifically projected for the training of motor skills specifically virtual fixtures. The HDS is an integrated system that merges haptic functionalities and Video Display Terminal (VDT) systems into one. It has been designed to provide visual and haptic perceptual information in order to avoid high mental loads and enable natural interaction with the user. Two applications to assist to human users to develop their motor skills to draw simple sketches were developed. The basic idea is generate a virtual template trajectory that the user must follow assisted with the force feedback capabilities of the HDS. In the first application, the virtual templates are generated from a given image file using image processing techniques, the templates are obtained and sent to the HDS controller which in combination with a graphical user interface (GUI) permits to the user to "fill" (follow) the virtual template. In the second application the templates are generated in a more dynamic way; the basic idea is to build a sketch using a set of virtual geometrical templates (VGT). The application currently allows drawing three types of VGTs: circles, lines and arcs. The user decides when and where request the assistance of the HDS to drawn a geometric shape. Based on the results we have demonstrated that the use of haptic interfaces improves and accelerates the acquiring of sketching skills.

\section{Haptic desktop}

The Haptic Desktop System (HDS) developed at PERCRO is our multimodal interface solution for design simple sketches. In Figure 3 a conceptual representation of this interface is shown, the user can freely design sketches inside the workspace of the interface which correspond with the superficies of a screen, enabling to the interface render the haptic and visual feedback in the same point in the $2 \mathrm{D}$ space.

\subsection{Design guidelines}

Two types of design guidelines have been employed during the preliminary definition of the system: qualitative and performance guidelines. According to qualitative guidelines the device had to show ergonomic features, which make the use of the system very comfortable. These guidelines have regarded: the workplace, the quality of the visual feedback, the aesthetics of the system, and the modality of interaction. Another very important guideline is the reduction of mental load during the use of the device.

The system design and control should allow the user to feel and control haptic interaction just below his fingertips, while directly viewing the effects of his actions on the computer screen (co-location). Specific state of the art analyses [Jansson \& Öström, 2004] have verified that co-location greatly enhances the user performances in HCI while reducing the mental load of the interaction. The haptic device has therefore to be calibrated in order to collimate the position of the usage tool (end-effector), with the pointer within the computer screen. The accuracy provided in design, should ensure that the sensitivity of the system is far beyond the pixel resolution of the screen. 
These guidelines have steered the main design choices of the system: the presence of the haptic interface should minimally interfere with the visual feedback and therefore it was decided to make use of transparent materials for building; all cables and connection should be hidden to the user; the device should offer the possibility of using a common pen as an interaction tool or the possibility of changing the end part with different tools; the device should be able in any case to replace and substitute the mouse in all its basic functions (point, select, click,...); the kinematic of the device should be designed in order to not interfere with the user limbs, it should preferably move in the opposite space with respect the user; the device, whenever unused, has to be closable in order to left the desktop free.

According to performance guidelines the device should have the following characteristics: a comfortable workspace wide enough to allow user to interact in writing operation: the workspace estimated had to cover at least the same dimensions of a notebook $(270 \times 360 \mathrm{~mm})$; for design and writing related applications a typical position resolution well below $1 \mathrm{~mm}$ was identified. Backlashes were not allowed in kinematic design. A set of preliminary experiments, carried out with pens in writing-and-contour-following-tests suggested a target continuos force of about 1-1.5N. Similar tests outlined a maximum residual mass of $0.2 \mathrm{Kg}$.

A high isotropy of the mechanical parameters (manipulability ellipsoid $\sim 1$ ) was required all over the workspace in order to reduce the distortion effects and to maximize the exploitation of the motors. In order to achieve high control bandwidth and facilitate the system maintenance and development a high degree of integration with the hosting OS was required.

Finally, in order to reduce the cost related to the system manufacturing two features were required to the system design: simplicity and possibility of manufacturing with low precision (low cost) technologies such as the laser cutting..
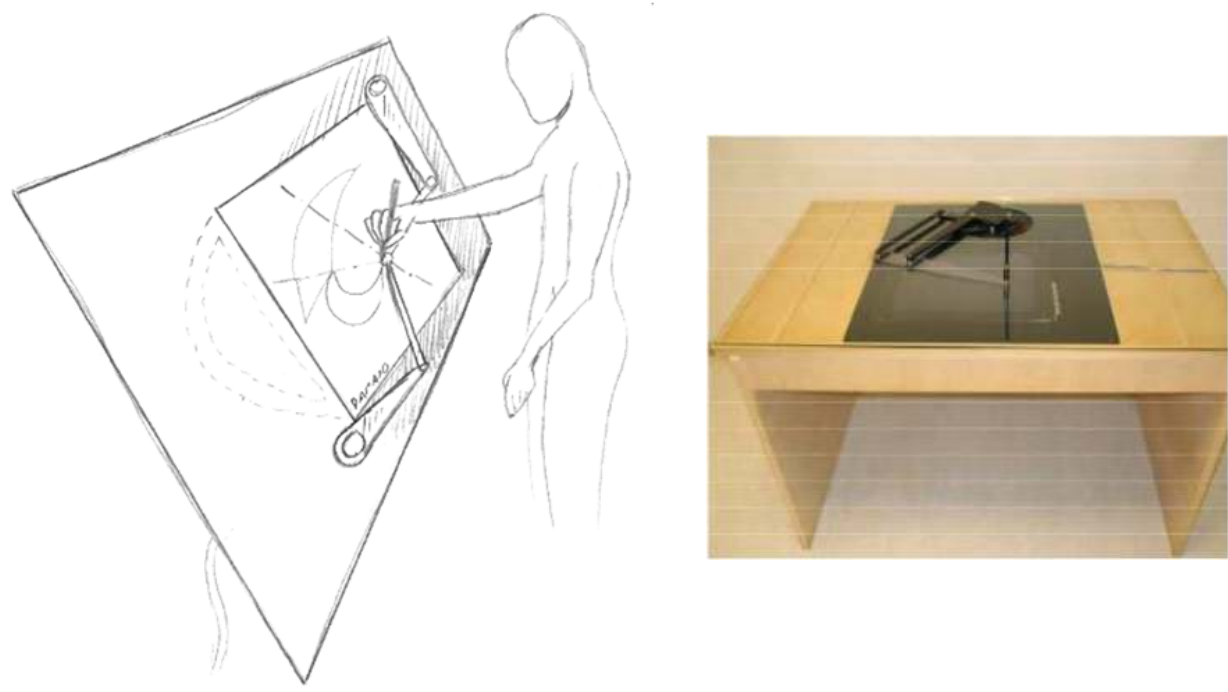

Fig. 3. The Concept and Implementation of the Haptic Desktop. 


\subsection{Design of the haptic device}

An LCD monitor of the proper size and resolution $34 \times 27 \mathrm{~cm}(1024 \times 768$ pixels $)$ was chosen and integrated within a desktop plane. The haptic device is a 2DOF system which employs and hybrid serial parallel kinematic structure. This solution behaves an end-effector, which slides over the monitor and desk plane in correspondence with the computer pointer. The specific kind of materials adopted has chosen in order to reduce the friction factor among surfaces.

In order to improve the performances of the haptic device (transparency, manipulability, workspaces, reflected mass), the following solutions to the design guidelines mentioned previously have been adopted:

- Both actuators have been grounded and attached to the base link. As in parallel manipulators, the grounding of the motors allows to reduce the amount of movable masses and to increase the overall stiffness. The specific serial parallel design was used for transmitting the actuation to the end effector and to solve the typical workspace limitations of parallel devices (Frisoli, Prisco, Salsedo, \& Bergamasco, 1999);

- Brushed DC motors have been selected: the amount of target force in this kind of applications is limited to few newtons; therefore the iron-less construction of the rotor allows the best compromise of performances.

- Capstans and metallic in tension tendons have been used as means of transmission of forces from the actuators to the joints. This solution allows completely avoiding geared transmission for the reduction of the transmission ratio, and therefore to avoid the backlash and friction issues related to this kind of solutions.

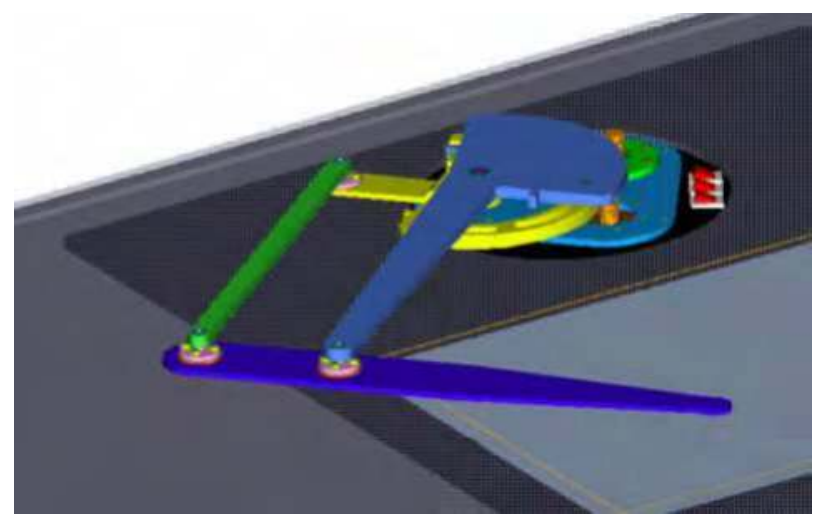

Fig. 4. Assonometric view of the haptic interface.

The structural parts have been realized with light materials (aluminium, plastic) and the specific design used allows the system to correctly operate even in presence of medium tolerance manufacturing.

The whole system has been designed to be integrated with the work-plane of a desk: the computing unit, the power supply, the motors and the electronics for the control of the haptic interface have been placed under the desktop so that the desk plane is completely free and the operator has direct access only to the visual and haptic systems. 
Figure 4 shows an assonometric view of the design prototype: in this system, on the desk plane only the required mobile parts of the interface are present; a new design of the prototype allows moving event the capstans and motor pulleys below desk plane. Three buttons have been left near the haptic base in order to control the computing-unit power, to enable force feedback, and to switch on/off the desk light.

\subsection{Optimization of the design}

In order to evaluate the proper length of link 1 and link 2, a preliminary analysis was set. As basic assumption we required that the device should cover the whole workspace of the LCD while maintaining a good factor in the manipulability ellipsoid. The variation of the condition number of the manipulability ellipsoid (while the system moves over the workspace) was considered in this design phase. A script procedure was initially set up to estimate the conditioning number all over the workspace when links' sizes and interface position were changed.

In Figure 5, the best-case solution is graphically represented. The workspace areas have been coloured by the value of the condition number (c). The colour type changes at each tenth of unit. Central area has values between 0.9 and 1 , second area values range between 0.8 and 0.9 and the third area values range between 0.7 and 0.8 . More than $90 \%$ of the overall workspace has $\mathrm{c}>0.9$.

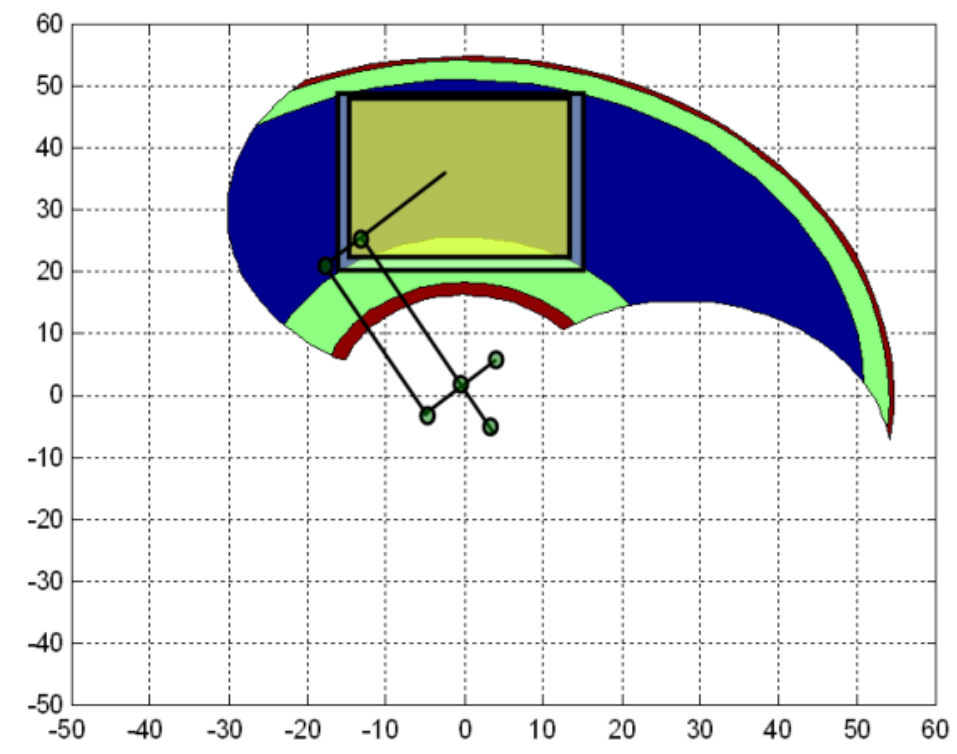

Fig. 5. Workspace evaluation

In Figure 6, the haptic desktop workspace in case of link 1 length equal to $300 \mathrm{~mm}$ and link 2 length equal to $270 \mathrm{~mm}$ is shown. Two capstans are used for reducing the inertia of moving masses and for introducing a reduction ratio. These reduction ratios have been dimensioned to obtain a condition number in the middle of the workspace equal to 1. 


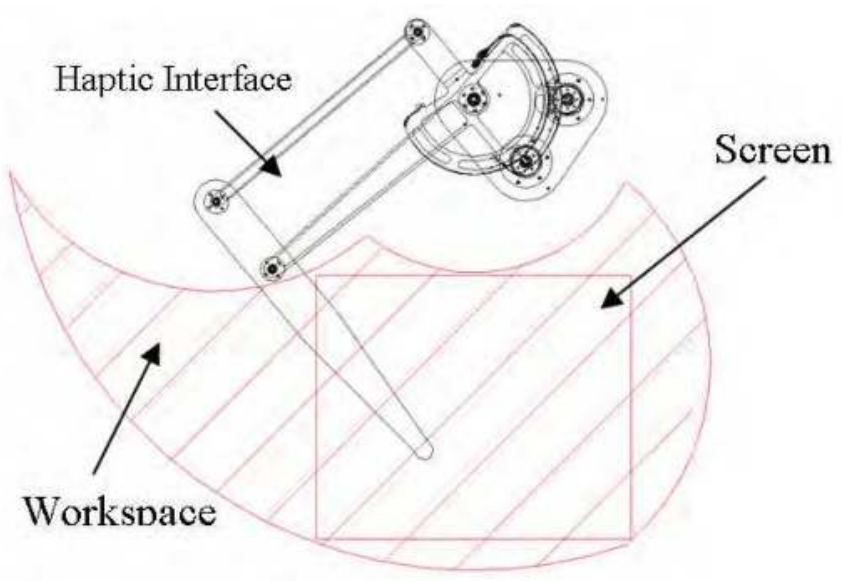

Fig. 6. Workspace of the haptic interface.

\subsection{Components choice}

The kinematics of the haptic interface has been determined according to the chosen link lengths and describe end-effector's coordinates referred to the central pivoting joint (Figure 7).

$$
\begin{aligned}
& X_{e e}=-L_{2} \cdot \cos \left(q_{2}\right)+L_{1} \cdot \cos \left(q_{1}\right) \\
& Y_{e e}=-L_{2} \cdot \sin \left(q_{2}\right)+L_{1} \cdot \sin \left(q_{1}\right)
\end{aligned}
$$

From (1) and (2) $L_{1}$ and $L_{2}$ are the lengths of link 1 and link 2 while $q_{1}$ and $q_{2}$ are the angles referred to the joints. On the other hand, the end-effector's coordinates referred to the motors, $q_{1}$ and $q_{2}$ can be computed as follows:

$$
\begin{gathered}
q_{1}=\frac{r_{1}}{R_{1}} \cdot \theta_{1} \\
q_{2}=-\frac{r_{2}}{R_{2}} \cdot \theta_{2}
\end{gathered}
$$

From (3) and (4) are the rotations of the two actuators, $r_{1}$ and $r_{2}$ are the radii of motor pulleys and $R_{1}$ and $R_{2}$ are the radii of the capstans. Using the above relationships it was possible to identify the commercial components to be used within the haptic device (motor type and size, sensors, etc). The homogeneity of the conditioning number has allowed us to size out the design by using the workspace centre as reference point. Maxon motor $3557024 \mathrm{CR}$ was used for actuation. With this solution the Haptic Interface can generate on user's hand up to $3 \mathrm{~N}$ of continuous forces and $5 \mathrm{~N}$ of peak forces. To detect device motion 1024 counter per cycle optical encoders where adopted with a $4 \mathrm{X}$ decoding. Such a choice leads to a spatial sensitivity of about $30 \mu \mathrm{m}$ at workspace centre (pixel size is about $300 \mu \mathrm{m}$ ). 
The design of the hardware system is characterized by its compactness and low cost. The HD is composed by a Single Board Computer (SBC) with Windows XP, a LCD screen and a Haptic Interface actuated by two DC motors (Figure 8).

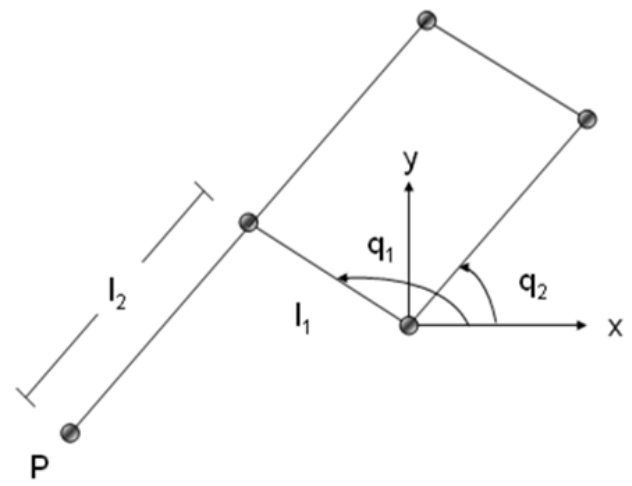

Fig. 7. Kinematics of the system

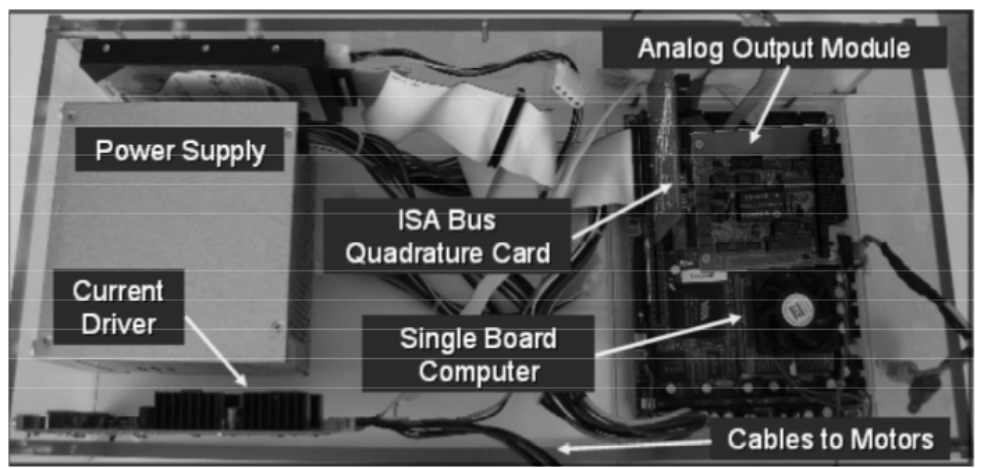

Fig. 8. Hardware unit has been designed to fit into a small acrylic box.

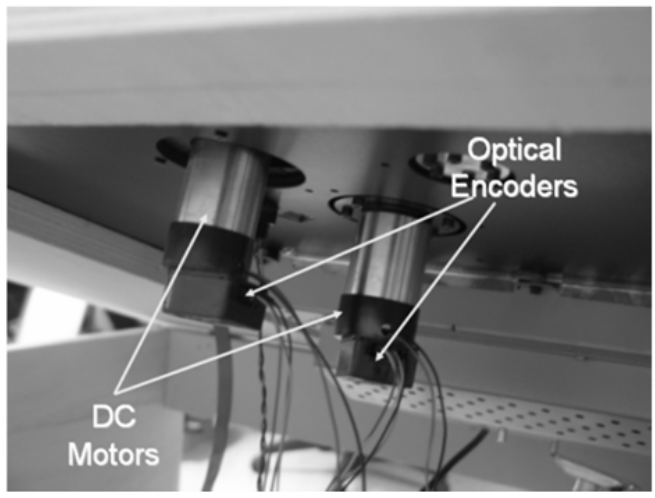

Fig. 9. The DC Motors and Optical Encoders are mounted on the rear of the desk. 
The encoder signal is read using an ISA Bus Quadrature Encoder Card attached to the SBC. The current to motors is generated by using a PC/104 module with two channel analog outputs (AX10415) and a custom-made current driver which is fed by the power supply of the computer (Figure 9). The Figure 10 shows the system's architecture.

\subsection{Control system}

The base control system of the HD has been designed and implemented with Matlab ${ }^{\mathrm{TM}}$ Version 6.5 and Simulink Version 5.0. Due to the restrictions of Windows operating system to execute "hard" real-time applications; the Matlab's Real-time Workshop provides two solutions to implement the real-time controller without adding additional hardware to the system: Real-Time Windows Target (RTWIN) and Generic Real-Time Target (GRT).

The Real-Time Windows Target uses a small real-time kernel to ensure the real-time application runs in real time. The real-time kernel runs at CPU ring zero (privileged or kernel mode) and uses the built-in PC clock as its primary source of time. In order to share the data from the Real-Time Windows Target kernel to the outside world (i.e. graphical system), an Sfunction was written as a SimViewing device which lets the creation of a shared memory by means of a static library. Such S-function does not compile into real-time code but acts as a Simulink External mode interface instead. This is due under the real-time kernel, the Win32 API cannot be executed as a component of the real-time application. One drawback of this implementation is that the updating time of the data send to the external application is too low, although the real-time control loop can achieve frequencies up to $2 \mathrm{k} \mathrm{Hz}$.
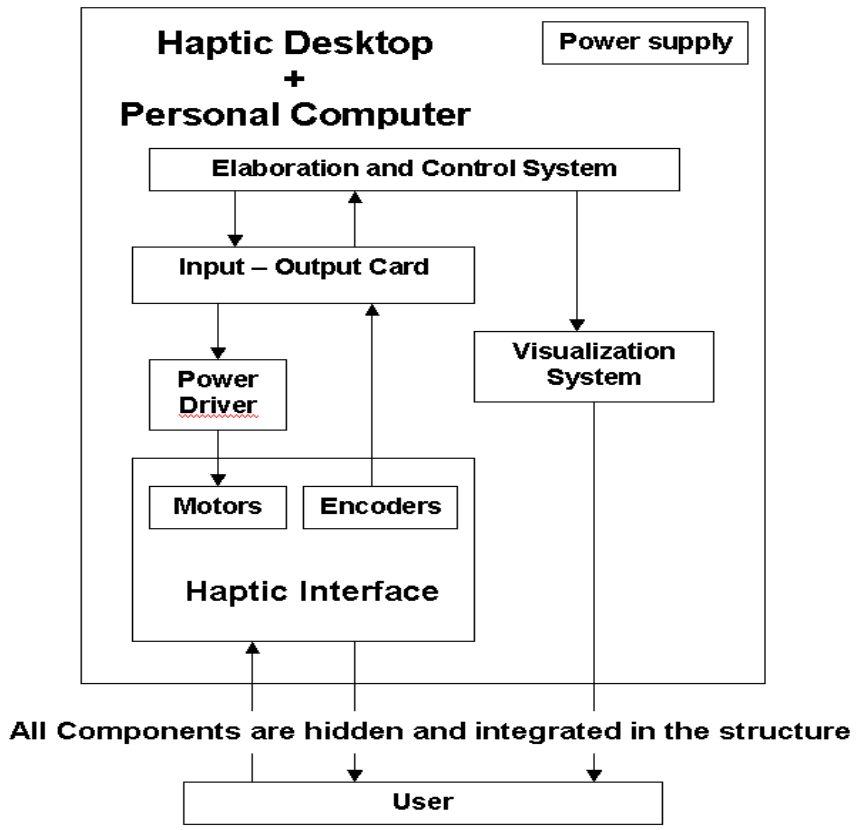

Fig. 10. Architecture of the system. 
On the other hand, the Real-Time Workshop provides a generic real-time development target. The generic real-time (GRT) target provides an environment for simulating fixed-step models in single or multitasking mode. A program generated with the GRT target runs the control model as a stand-alone program under the computer. The real-time execution is achieved by controlling the real-time clock using the multimedia timers (such capability can be done by modifying the grt_main.c file). Such timers have the highest resolution under the Windows OS, with a minimum resolution of 1 millisecond. The data is shared with the graphical application also by a static library (Figure 11).

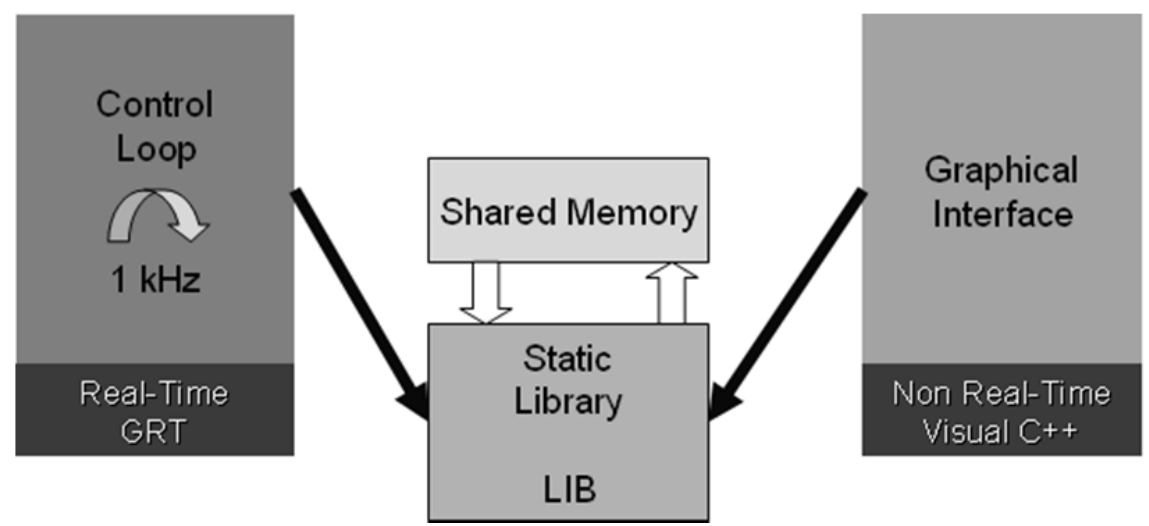

Fig. 11. Shared memory is managed by means of a static library

A calibration phase of the $\mathrm{HI}$ has been implemented to measure correctly the joint positions so that we can assure the coincidence between the haptic and graphical information. This phase consists on moving both links, using a velocity control, into a well know configuration (Figure 12), where the difference between the real joint position $\left(q_{1}=130^{\circ}\right.$ and $\mathrm{q}_{2}=170^{\circ}$ ) and the measured joint position is computed and used to correct the signal coming from the encoders.

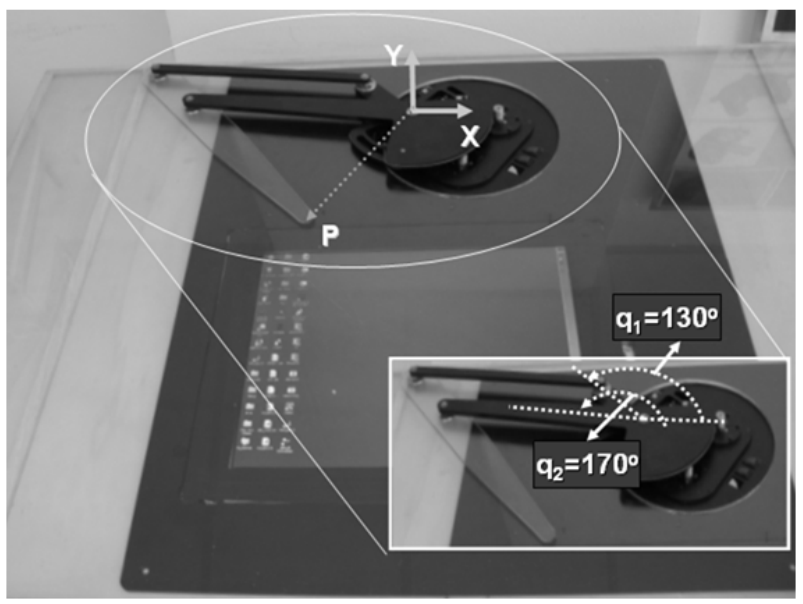

Fig. 12. The reset position for calibrating the $\mathrm{HI}$ is shown. 
In the case of the GRT, the I/O ports cannot be accessed directly, due to the restrictions of the OS. This problem can be solved by using the PortTalk utility which allows the permissions to the real-time code to access the I/O ports. Therefore, at this moment, we have implemented the base control by compiling the system model under the generic realtime target, with a sampling frequency of $1 \mathrm{k} \mathrm{Hz}$.

This control system was implemented on the same architecture of the target application system. This device was made by an embedded PC (PIV 1.4GHz 1 GB RAM). Specific I/O board for detecting the encoder position and for generating the DAC command to be forwarded to drivers where implemented on the ISA and PC104 busses offered by the embedded board. The desktop backside was designed in order to host motors, power supply, hard disk, power drivers, mother boards and IO boards, all within a minimal regular square box as shown in Figure 9. The high integration achieved has allowed us to implement all control procedures and drivers within the hosting OS kernel (Windows XP).

Several functional control solutions are available to use the system: a basic mouse replacement toolkit allow to make use of the system interface to control the pointer position within the screen and interact to the GUI; it is also possible to directly control the hardware device by software and or control CAD; a set of functionalities libraries also implement main feature to facilitating integration within application.

\subsection{Graphical system}

In order to access the resources of the operating system, the mouse functionalities have been replaced by computing the end-effector position and by reading the signals coming from the buttons attached to the HD. Just after the real-time control is started, the system cursor is controlled, by using the mouse Win32 APIs, so that the contact point position is used to refresh cursor's position.
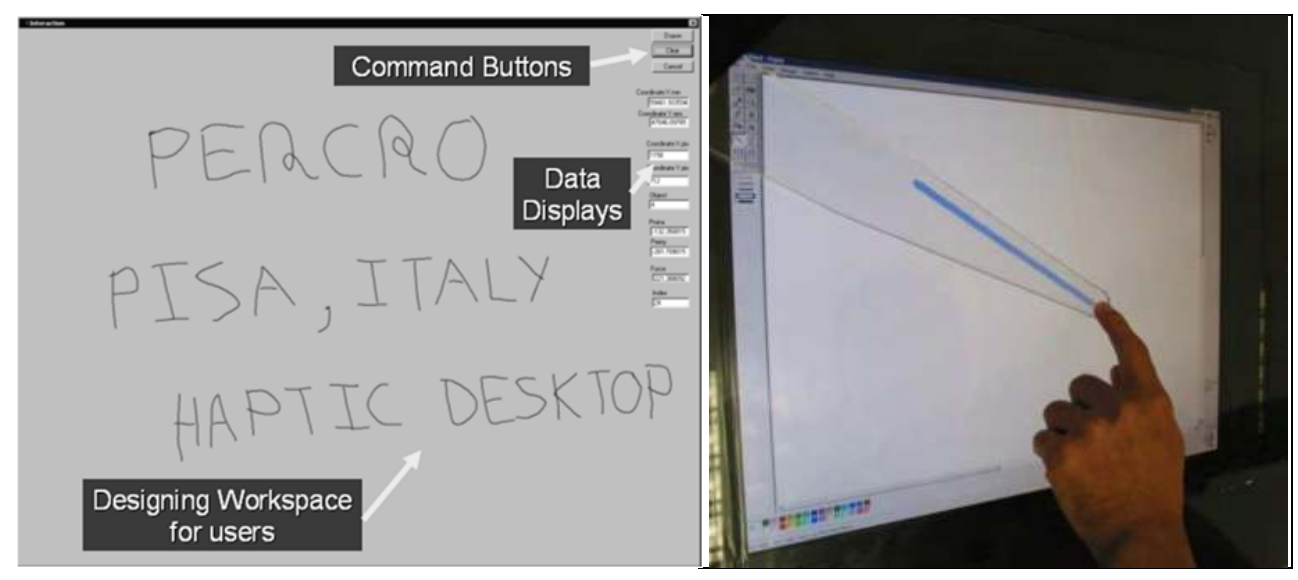

Fig. 13. The drawing interface window is shown.

As it was explained before, the contact position is computed by the real-time application and then, it is transferred to the shared memory. Then, the non-real time application developed with Visual $\mathrm{C}++$, obtains the position and transform it to screen coordinates. 
Furthermore, the non-real time application displays an interactive drawing window where the user can draw any kind of shapes or write words by changing grasping the $\mathrm{HI}$ and by controlling the command buttons (Figure 13).

\section{A transfer skill system for teaching how to draw to unskilled persons}

As mentioned previously, the core idea is to use the HDS as a tool capable of interacting dynamically with the human on the basis of the action he decides to perform. The use of an automated system in the skill transfer process has several advantages: the process could be repeated indefinitely with a high level of precision, time and location flexibility, the user can improve its performance without the assistance of a teacher and his progress can be evaluated numerically through automated procedures.

The HDS aims to be an interactive bi-directional skill transfer system that can emulate the presence of a human assistant, guiding the user movements on the trajectories chosen by the user himself (Figure 14).
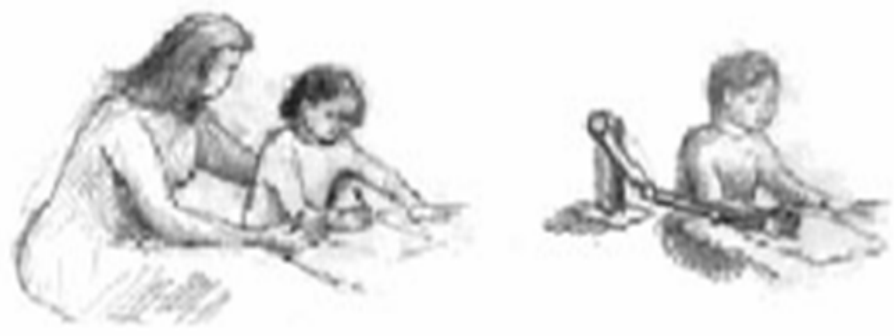

Fig. 14. Haptic Interfaces as assistant of abilities

In our previous research (Solis et al., 2002) it has been demonstrated the influence that proportional feedback programmed into haptic interface (HI) can have in the development of motor skills, in spite of this experiment was neither co-located nor coherent with the visual stimuli and only could assist to drawn a circle with fixed radio. The HD is our multimodal interface solution for teaches to design simple sketches.

We have developed two applications where the HDS assists the users in the sketching of simple drawings. The user interacts with the system through different means of feedback (visual, force, audio).

\subsection{Assisting to sketch using fixed templates}

In order to present fixed templates to the users, we have developed an application that transforms the principal edges of a given input image file (jpg, bmp, tiff, etc.) into trajectories for later HDS interpretation, this way the "trajectories" are generated without any kind pre programming. The first process applied to the image is a median filter to remove the possible noise present, then a equalization and binarization is done (Pratt, 1991), after that, to detect its edges a Canny edge detector (Canny, 1986) is used, then the resulting 
edges are s segmented using label connected components in a binary image (Haralick \& Shapiro, 1992), subsequently its components that are not more longer than a minimum predefined size are erased. The remaining components are converted to one pixel wide curves applying on them the morphological thin operator (Haralick \& Shapiro, 1992).

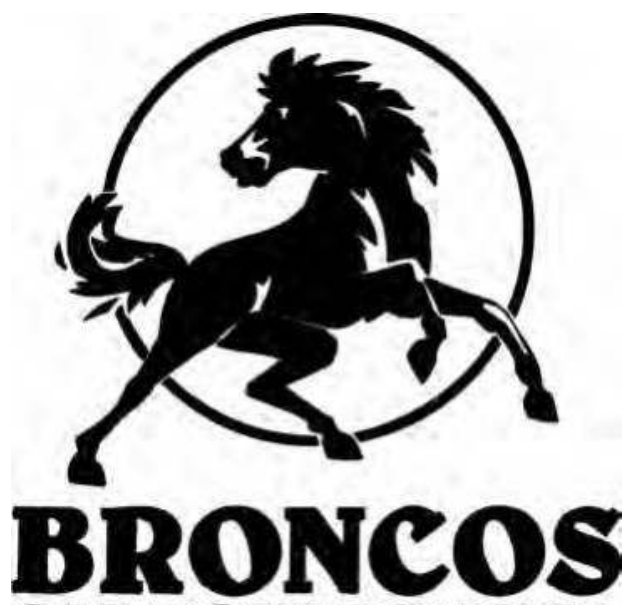

a) Original image file

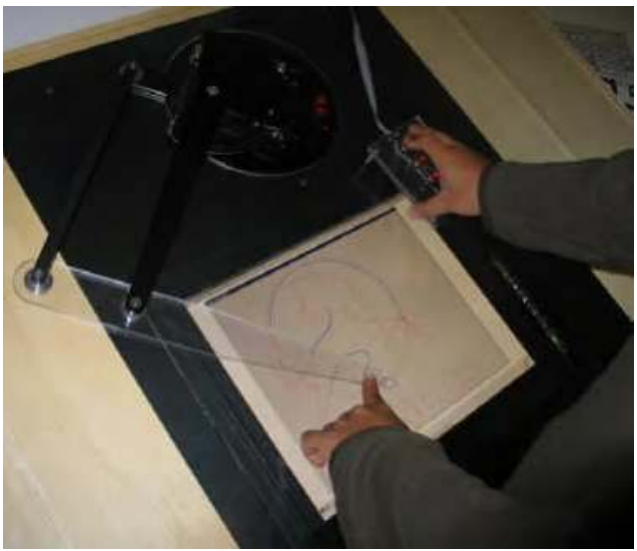

c) Haptic and Visual Feedback

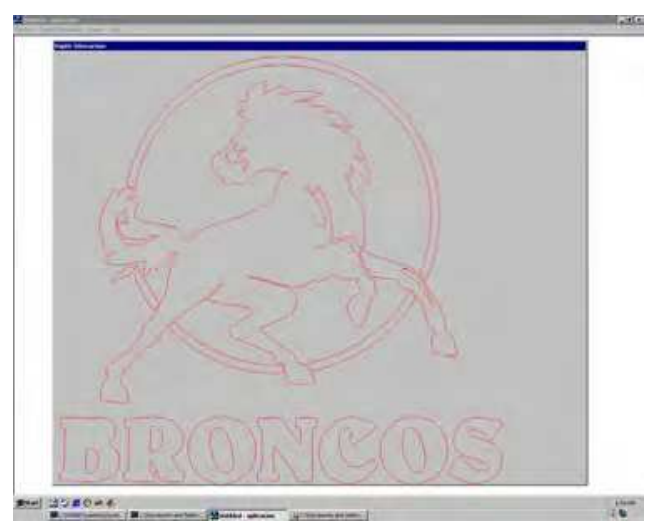

b) Processed Image "Trajectories"

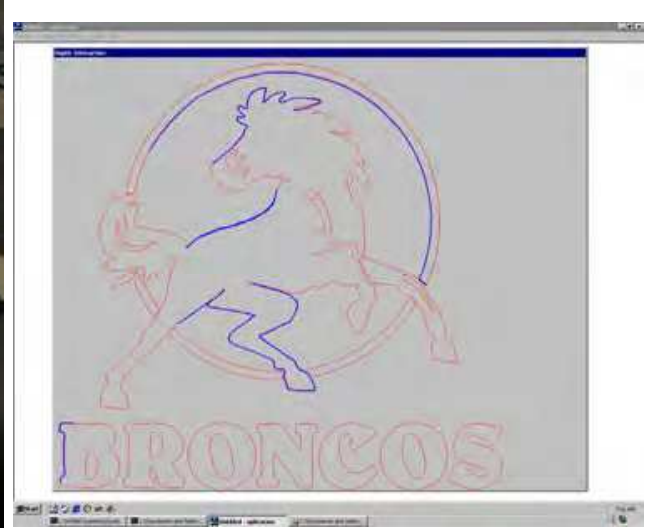

d) Visual Feedback

Fig. 15. The HDS live a virtual tutor.

Finally, the coordinates of the remaining objects (virtual templates) are converted to the HDS reference system controller and stored in a segment memory, which is shared with the HDS controller. The Figure 15a shows an example of original jpg image and Figure 15b shows the results of the image processing.

The GUI displays the processed images and assists to the user to interact with the templates. The application is controlled through four buttons, the first two (top to bottom, Figure 15c) acts like the left and right mouse buttons, the third cleans the screen and the fourth toggles the 
force feedback. The user can move the HDS's end effector (HEE) freely in all its workspace until he presses the first button. On button press, a blue dot is drawn in the end effector position (visual feedback) and a collision detection algorithm looks for collisions between the HEE position and any template (trajectory) inside a radius of $40 \mathrm{~mm}$ from the HEE center.

If a collision is detected, the HDS responds using force feedback to maintain the HEE constrained to the nearest point of the contacted template. Force feedback is generated with the following control law:

$$
F=-K_{P} \Delta-K_{v} \dot{\Delta}
$$

Where $\Delta$ is the distance between the HEE Position and its nearest point on the template, and $K_{\mathrm{P}}$ and $K_{\mathrm{v}}$ should be small enough to allow a free movement along the trajectory while creating a force feedback outside the trajectory, its values where find experimentally. When the HDS exerts a force of two Newtons or more, the user receives an auditory feedback.

Figure 15c shows a user interaction with the application: it is possible to see the coherence and colocation between haptic and graphical information. The visual feedback presented to the user is shown close up in Figure 15d.

\subsection{Assisting to sketch using interactive templates}

A second application was developed in order to assist to drawn sketches interactively, the HDS's audio feedback, coherence and co-location between haptic and graphical information are exploited. The GUI developed is minimalist and simple to use (Figure 16). At the moment it can assist to design three different types of virtual geometrical templates (VGTs): straight line, circle and arc. The use of the buttons in order to control the application, the collision detection algorithm and the auditory cues have the same behaviour, that its fixed template counterpart.

The user can move the HEE freely in all the workspace and drawing a free sketch when the first button is pressed. When the user decided to drawn a VGT with the assistance of the HDS, he first, must indicate the VGT's first point, pressing the second button and choosing of a menu the VGT that he want drawn (Figure 16a); this first point could be; a) the beginning of a straight line, b) beginning of an arc or c) the center of a circle. Once that the first point has been selected, he must move the HEE to another position inside the GUI and with the first button must to mark the second and third points depending on which VGT has been selected. The second point is the end of the straight line if a line VGT has been chosen, on other hand if circle VGT was selected, the second point is used to calculate the radius respect to the first point and then generate a circumference around it. If arc VGT is chosen, the three points marked are united interpolating a second order spline curve.

Once that all the control points have been defined, the application draws a virtual "template trajectory" (Figure 16b), after that, the user can move the HDS's end effector (HEE) freely in all its workspace until he presses the first button. On button press, a blue dot is drawn in the end effector position (visual feedback) and a collision detection algorithm looks for collisions between the HEE position and the virtual template. Similarly to the fixed template application, the HDS maintains its HEE constrained to the nearest point of the contacted template and draws a blue line. 


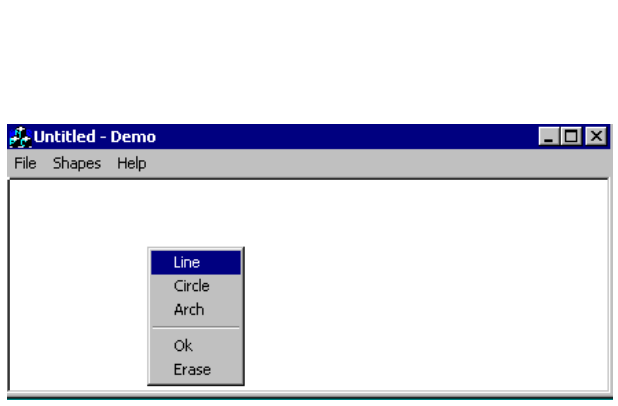

(a) Choosing between three different geometric primitives (straight line, circle and arc).

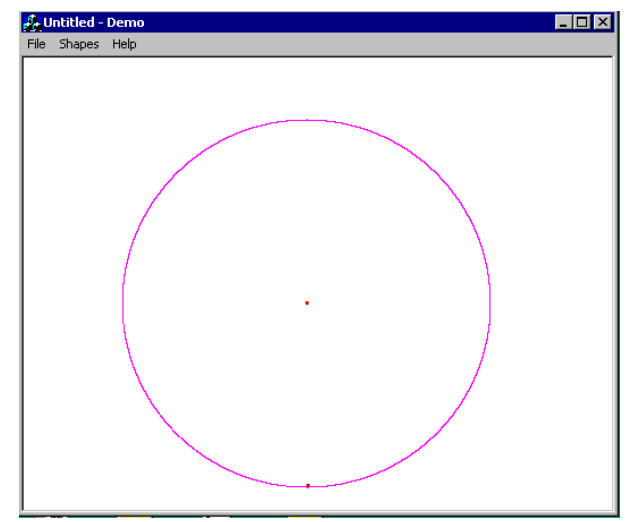

(b) A circle "template"

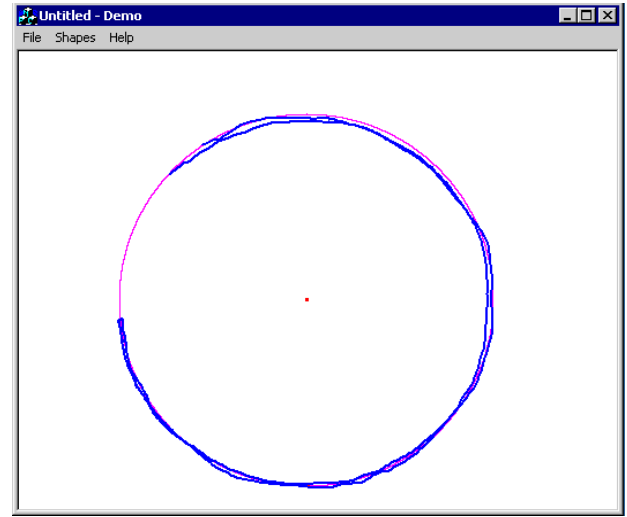

(c) The user "fills" the template

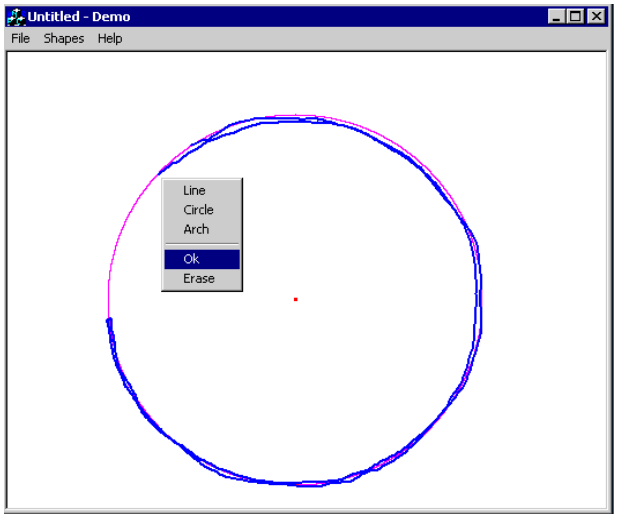

(d) The user decides to finalize with assistance of the HDS

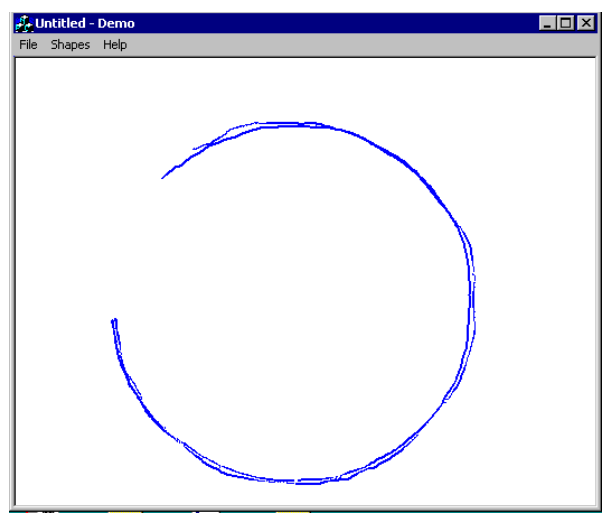

(e) Final sketch without "template"

Fig. 16. Interaction with the HDS assistant. 
When the user desires finalize with the HDS's assistance, he must press the second button and indicates using a menu, if erase the line recently drawn or maintains it; if he decides maintain it, the virtual template will be erased and only the line drawn made remains (Figure 16.e). In order to design more complex sketches is necessary repeat all the precedent steps and depending of the ability of the user can draw more complex design.

\section{Experiment to validate sketching skills for fixed templates}

To check that the users learn sketching faster when they are provided with force feedback, we carried out the following test: The image used in the experiment is shown in the Figure 17b, which is based on the original image shown in the Figure 17a. The test image was chosen due it has the basic components of more complex designs (lines, curves, circles, squares, etc).

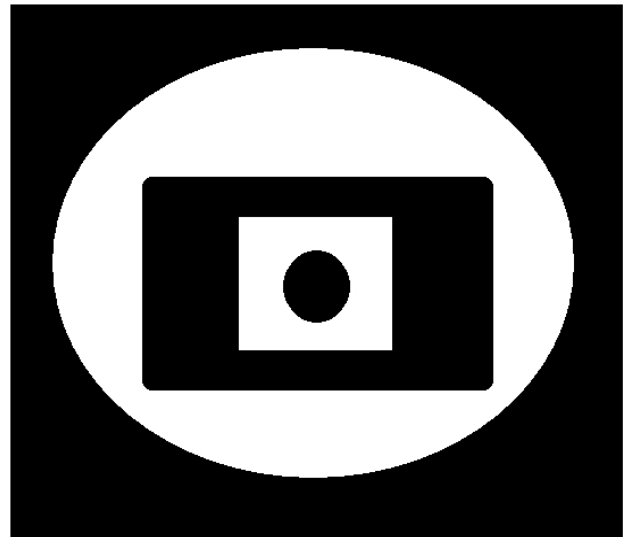

(a) Original

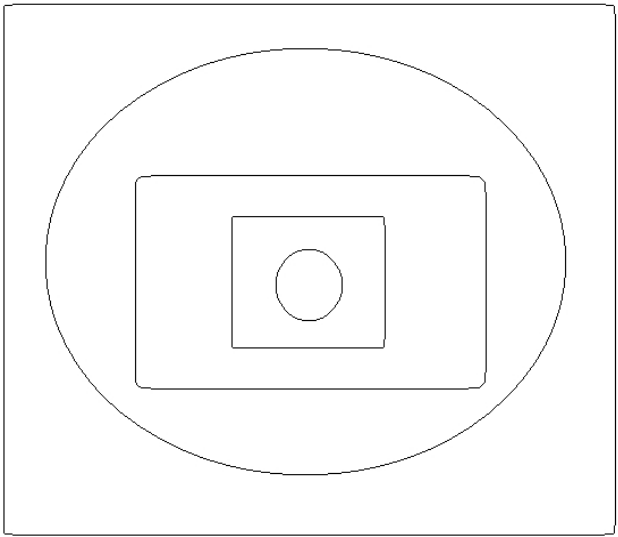

(b) Processed Image

Fig. 17. Test Image.

We requested to 10 users to trace the test image seven times as precisely as possible in terms of position. Since this task should model free drawing, no restriction on completion time or effector velocity was imposed. The users were divided in two groups of 5 members. At the beginning of each experiment, the users had five minutes for drawing on the test image in order to become familiar with the HDS. The users of the first group were stimulated only with visual feedback throughout the experiment. The users of the second group were stimulated with visual feedback on every trial, and they received the force feedback only in the second, fourth and sixth trials.

We used the performance parameter described by (6) to measure if user learning of drawing skills was indeed accelerated. The PSD parameter involves the time, velocity, distance and error (between the HEE Position and the point on the trajectory nearest to the HEE) when the user is drawing.

$$
P_{S D}=\frac{\int_{0}^{t}|\Delta| d t}{L=\int_{0}^{t} v d t}
$$




\subsection{Results}

In the Figure 18, we present the normalized sum of PSD over the subjects of the two groups: the PSD sum for trial 1 has been made equal to 1.

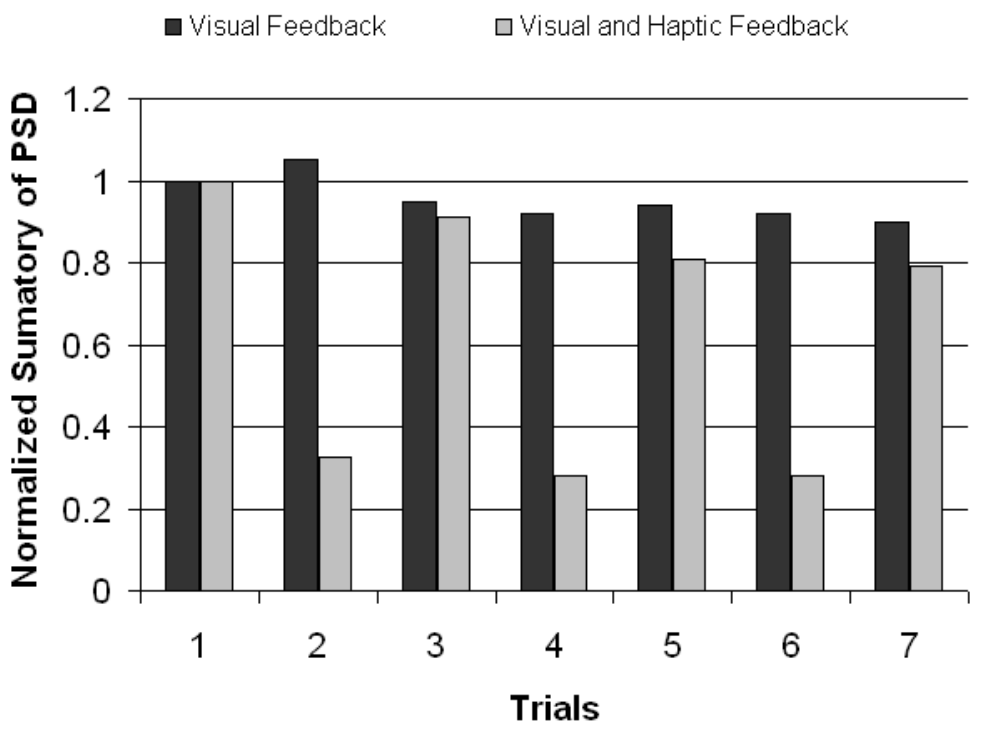

Fig. 18. Evolution of normalized sumatory of the PSD

We can obviously observe a strong difference between performances on trial 2, 4 and 6 . What is more interesting, we can observe that for the Visual Feedback Only group, learning stops at Trial 4, while the Visual Feedback and Haptics group continues learning to Trail 7. Furthermore, at trial 7 the normalized sum of PSD is lower for the Visual Feedback and Haptics group, which leads us to conclude (within the limits of a small scale experiment with a small number of subjects) that Haptic feedback enables learning for a longer time and leads to better results for the task at hand.

We didn't validate the improvement of the user to drawn simple skills due there aren't restrictions about position, size of the sketch's traces. It is our first attempt to develop a more advanced assistant, which will have more virtual geometric templates and the auditory cues will be exploited more properly, i.e. vocal instructions.

\section{Conclusions}

The efficacy of the Haptic Desktop to transfer basic skills to drawn simples sketches was demonstrated through the use of two applications, in both the HD acts like a virtual teacher that uses its force feedback and collocated capabilities to retain the cursor's position (controlled by the end effector position) in contact the borders of predefined sketch. The sketch could be either fixed or interactive, the fixed ones are generated from images, and the interactive ones are generated by the user whom can decide where, how big and which primitive geometric element draw (circle, line or arch). 
The experiment described in this chapter, showed that the use of force and visual stimuli helps to the user to drawn the simple sketches more quickly and with less errors, but more work must be done in order to enable to the interface to understand when and how the feedback stimuli have to be generated, to do it, it would be necessary obtain velocities and forces (magnitude and orientation) of the hand users and develop advanced algorithms that recognize the difference between drawn a primitive element as expert or novice. These algorithms are difficult to develop and possibly the variables to quantify the quality of the hand movement could be not enough.

\section{References}

Annelise Mark Pejtersen, J. R. (1997). Ecological Information Systems and Support of Learning: Coupling Work Domain Information to user Characteristics. Handbook of Human-Computer Interaction. North/Holland.

Flach, J. M. (1994). Beyond the servomechanism: Implications of closed-loop, adaptive couplings for modeling human-machine systems. Symposium on Human Interaction with Complex Systems. North Carolina AET State University.

Frisoli, A., Prisco, M., Salsedo, F., \& Bergamasco, M. (1999). A two degrees-of-freedom planar haptic interface with high kinematic isotropy" Robot and Human Interaction. 8th IEEE International Workshop on Robot and Human Interactive Communication, (p. 297 - 302).

Gopher, D. (2004). Control processes in the formation of task units. 28th International Congress of Psychology. Beijing, China.

Haralick, R., \& Shapiro, L. (1992). Computer and Robot Vision (Vol. I). Addison-Wesley.

Jansson, G. J., \& Öström, M. (2004). The Effects of Co-location of Visual and Haptic Space on Judgments of Form. EuroHaptics.

McCullough, M. (1999). Abstracting Craft. MIT Press.

M. Chignell, P., \& Takeshit, H. (1999). Human-Computer Interaction: The psychology of augmented human behavior. In P. Hancock (A cura di), Human performance and Ergonomics. Academic Press.

Norman, D. (1986). User-centered systems design. Hillsdale.

Norman, D. (1988). The design of everyday things. New York: Basic Books.

Pratt, W. (1991). Digital Image Processing. John Wiley \& Sons, Inc.

Solis, J., Avizzano, C., \& Bergamasco, M. (2002). Teaching to Write Japanese Characters using an Haptic Interface. In Proceedings of the 10th International Symposium on Haptic Interfaces for Virtual Environment and Teleoperator Systems. Orlando, FL. 


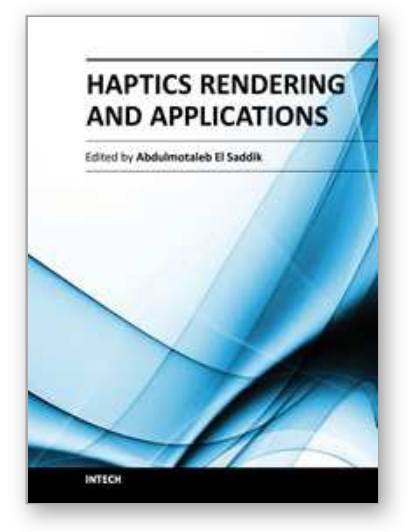

\author{
Haptics Rendering and Applications \\ Edited by Dr. Abdulmotaleb El Saddik
}

ISBN 978-953-307-897-7

Hard cover, 246 pages

Publisher InTech

Published online 27, January, 2012

Published in print edition January, 2012

There has been significant progress in haptic technologies but the incorporation of haptics into virtual environments is still in its infancy. A wide range of the new society's human activities including communication, education, art, entertainment, commerce and science would forever change if we learned how to capture, manipulate and reproduce haptic sensory stimuli that are nearly indistinguishable from reality. For the field to move forward, many commercial and technological barriers need to be overcome. By rendering how objects feel through haptic technology, we communicate information that might reflect a desire to speak a physicallybased language that has never been explored before. Due to constant improvement in haptics technology and increasing levels of research into and development of haptics-related algorithms, protocols and devices, there is a belief that haptics technology has a promising future.

\title{
How to reference
}

In order to correctly reference this scholarly work, feel free to copy and paste the following:

Otniel Portillo-Rodriguez, Carlo Avizzano, Oscar Sandoval Gonzalez, Adriana Vilchis-Gonzalez, Mariel DavilaVilchis and Massimo Bergamasco (2012). Training Motor Skills Using Haptic Interfaces, Haptics Rendering and Applications, Dr. Abdulmotaleb El Saddik (Ed.), ISBN: 978-953-307-897-7, InTech, Available from: http://www.intechopen.com/books/haptics-rendering-and-applications/improving-motor-skills-using-hapticinterfaces

\section{INTECH}

open science | open minds

\author{
InTech Europe \\ University Campus STeP Ri \\ Slavka Krautzeka 83/A \\ 51000 Rijeka, Croatia \\ Phone: +385 (51) 770447 \\ Fax: +385 (51) 686166 \\ www.intechopen.com
}

\author{
InTech China \\ Unit 405, Office Block, Hotel Equatorial Shanghai \\ No.65, Yan An Road (West), Shanghai, 200040, China \\ 中国上海市延安西路65号上海国际贵都大饭店办公楼 405 单元 \\ Phone: +86-21-62489820 \\ Fax: $+86-21-62489821$
}


(C) 2012 The Author(s). Licensee IntechOpen. This is an open access article distributed under the terms of the Creative Commons Attribution 3.0 License, which permits unrestricted use, distribution, and reproduction in any medium, provided the original work is properly cited. 